\title{
On application of differential subordination for Carathéodory functions
}

\author{
V. Agnes Sagaya Judy Lavanya \\ Dr. MGR Janaki College of Arts and Science \\ Chennai 600028, TamilNadu, India \\ email: lavanyaravi06@gmail.com
}

M. P. Jeyaraman

L. N. Government College

Ponneri, Chennai 601 204,

Tamil Nadu, India

email: jeyaraman_mp@yahoo.co.in
H. Aaisha Farzana

A. M. Jain College

Meenambakkam, Chennai 600 114,

Tamil Nadu, India

email: h.aaisha@gmail.com

\begin{abstract}
New sufficient conditions involving the properties of analytic functions to belong to the class of Carathéodory functions are investigated. Certain univalence and starlikeness conditions are deduced as special cases of main results.
\end{abstract}

\section{Introduction}

Let $\mathcal{H}$ be the class of analytic functions in the open unit disk $\mathbb{D}:=\{z \in$ $\mathbb{C}:|z|<1\}$. Let $\mathcal{A}$ denote the class of all the functions $\mathrm{f} \in \mathcal{H}$ that satisfy the normalization $\mathrm{f}(0)=0, \mathrm{f}^{\prime}(0)=1$. Let $\mathcal{S}$ denote the subclass of $\mathcal{A}$ consisting of univalent functions. The function $f \in \mathcal{A}$ satisfying the conditions $\operatorname{Re}\left\{z f^{\prime}(z) / f(z)\right\}>0, \operatorname{Re}\left\{1+z f^{\prime \prime}(z) / f^{\prime}(z)\right\}>0$ belong to the familiar classes of starlike and convex functions denoted by $\mathcal{S}^{*}$ and $\mathcal{C}$ respectively. Let $f$ and $g$ be analytic in $\mathbb{D}$, then we say that $f$ is subordinate to $g$ in $\mathbb{D}$ (written $f \prec g$ )

2010 Mathematics Subject Classification: 30C45,30C 80

Key words and phrases: analytic functions, differential subordination, Carathéodory function, starlike function, univalent function 
if there exists a Schwarz function $w(z)$,analytic in $\mathbb{D}$ with $w(0)=0$ such that $f(z)=g(w(z)),(z \in \mathbb{D})$. In particular, if the function $g$ is univalent in $\mathbb{D}$, then the subordination is equivalent to $f(0)=g(0)$ or $f(\mathbb{D}) \subset g(\mathbb{D})$. Let us denote by $\mathcal{Q}$ the set of functions $q$ that are analytic and injective on $\overline{\mathbb{D}} \backslash \mathrm{E}(\mathrm{q})$, where $E(q)=\left\{\zeta \in \partial \mathbb{D}: \lim _{z \rightarrow \zeta} \mathbf{q}(z)=\infty\right\}$, and are such that $\mathbf{q}^{\prime}(\zeta) \neq 0$ for $\zeta \in \partial \mathbb{D} \backslash \mathrm{E}(\mathrm{q})$. Further, the subclass of $\mathcal{Q}$ for which $\mathrm{q}(0)=\mathrm{a}$ be denoted by $\mathcal{Q}(\mathrm{a})$. Let $\mathcal{P}(\alpha)$ be a class of functions of the form $p(z)=1+\sum_{n=1}^{\infty} p_{n} z^{n}$, which are analytic in $\mathbb{D}$, we say that $p(z) \in \mathcal{P}(\alpha)$ if $\operatorname{Re}\{p(z)\}>\alpha$. We note that for $\mathcal{P}(0):=\mathcal{P}$ is the class of Carathéodory functions in $\mathbb{D}$.

The function $\mathrm{q}_{\mathrm{c}}(z)=\sqrt{1+c z}$, maps $\mathbb{D}$ onto a set which is bounded by the lemniscate of Bernoulli. That is, $\mathrm{q}_{\mathrm{c}}(\mathbb{D})=\left\{w \in \mathbb{C}:\left|w^{2}-1\right|<\mathrm{c}\right\}$, and the class $\mathcal{S}^{*}\left(\mathrm{q}_{\mathrm{c}}\right)$ given by $\mathcal{S}^{*}\left(\mathrm{q}_{\mathrm{c}}\right)=\left\{\mathrm{f} \in \mathcal{A}:\left|\left(z \mathrm{f}^{\prime}(z) / \mathrm{f}(z)\right)^{2}-1\right|<\mathrm{c}\right\} \quad(0<\mathrm{c} \leq 1)$, has been briefly discussed in [17]. We consider the class $\mathcal{U}(\lambda)$ of analytic functions satisfying the following condition, $\mathcal{U}(\lambda):=\left\{f \in \mathcal{A}:\left|(z / f(z))^{2} f^{\prime}(z)-1\right|<\right.$ $\lambda, \quad 0<\lambda \leq 1\}$. From [16] it is known that the functions in $\mathcal{U}(\lambda)$ are univalent if $0<\lambda \leq 1$, but not necessarily univalent if $\lambda>1$.

Various sufficient conditions for Carathéodory functions were studied by authors in $[5,6,11,12,13,14]$. Using differential subordination as a tool, authors in [13] and [14] obtained sufficient conditions for Carathéodory functions.Recently, Kim et al. [5] obtained sufficient conditions involving the argument of the function such that the function is Carathéodory. Motivated by the aforementioned works, in this paper various results involving analytic function to be Carathéodory are obtained and as a consequence, sufficient conditions for functions to belong to the classes $\mathcal{S}^{*}\left(\mathrm{q}_{\mathrm{c}}\right)$ and $\mathcal{U}(\lambda)$ are provided. The results thus obtained generalize and extend certain recent results.

\section{Main results}

To prove the main results we need the following Lemma.

Lemma 1 [4] Let $w$ be a non constant regular function in $\mathbb{D}$. If $|w|$ attains its maximum value on the circle $|z|=r<1$ at $z_{0}$, then

$$
z_{0} w^{\prime}\left(z_{0}\right)=k w\left(z_{0}\right)
$$

where $\mathrm{k} \geq 1$ is a real number.

Lemma $2[1,3]$ Let $\mathrm{q} \in \mathcal{Q}(\mathrm{a})$, and let $\mathrm{p}(z)=\mathrm{a}+\mathrm{a}_{\mathrm{n}} z^{\mathrm{n}}+\cdots$ be analytic in $\mathbb{D}$ with $\mathrm{p}(z) \not \equiv \mathrm{a}$ and $\mathrm{n} \geq 2$, if $\mathrm{p}$ is not subordinate to $\mathrm{q}$ then there exist points $z_{0}=r_{0} e^{i \theta_{0}} \in \mathbb{D}$ and $\zeta_{0} \in \partial \mathbb{D} \backslash \mathrm{E}(\mathrm{q})$ and an $\mathrm{m} \geq \mathrm{n}$ for which $\mathrm{p}\left(\mathbb{D}_{\mathrm{r}_{0}}\right) \subset \mathrm{q}(\mathbb{D})$, 
1. $p\left(z_{0}\right)=q\left(\zeta_{0}\right)$

2. $\operatorname{Re}\left\{\frac{\zeta_{0} \mathrm{q}^{\prime \prime}\left(\zeta_{0}\right)}{\mathrm{q}^{\prime}\left(\zeta_{0}\right)}\right\} \geq 0$ and $\left|\frac{z \mathrm{p}^{\prime}(z)}{\mathrm{q}^{\prime}(\zeta)}\right| \leq \mathrm{m}$

3. $z_{0} \mathrm{p}^{\prime}\left(z_{0}\right)=\mathrm{m} \zeta_{0} \mathrm{q}^{\prime}\left(\zeta_{0}\right)$

4. $\operatorname{Re}\left\{\frac{z_{0} \mathrm{p}^{\prime \prime}\left(z_{0}\right)}{\mathrm{p}^{\prime}\left(z_{0}\right)}+1\right\} \geq \mathrm{m} \operatorname{Re}\left\{\frac{\zeta_{0} \mathrm{q}^{\prime \prime}\left(\zeta_{0}\right)}{\mathrm{q}^{\prime}\left(\zeta_{0}\right)}+1\right\}$

5. $\operatorname{Re}\left\{\frac{z_{0}^{2} \mathrm{p}^{\prime \prime \prime}\left(z_{0}\right)}{\mathrm{p}^{\prime}\left(z_{0}\right)}\right\} \geq \mathrm{m}^{2} \operatorname{Re}\left\{\frac{\zeta_{0}^{2} \mathrm{q}^{\prime \prime \prime}\left(\zeta_{0}\right)}{\mathrm{q}^{\prime}\left(z_{0}\right)}\right\}$.

Theorem 1 Let $0 \leq \alpha<1,0<\lambda \leq 1, \beta, \gamma, \delta, \mu \in \mathbb{R}$. For an analytic function $\mathrm{p}$ defined in $\mathbb{D}$ with $\mathrm{p}(0)=1$, if

$\operatorname{Re}\left\{(p(z)-\alpha)^{\lambda}\left(\mu+\beta(p(z)-\alpha)+\frac{\gamma}{p(z)-\alpha}+\frac{\delta z p^{\prime}(z)}{p(z)-\alpha}\right)\right\}>g(\epsilon(\alpha, \lambda), \alpha, \lambda)$,

where

$$
\begin{aligned}
g(u, \alpha, \lambda)= & -u^{\lambda+1}\left(\beta+\frac{\delta}{2(1-\alpha)}\right) \sin \left(\frac{\lambda \pi}{2}\right)+\mu \cos \left(\frac{\lambda \pi}{2}\right) u^{\lambda} \\
& +\left(\gamma-\frac{\delta(1-\alpha)}{2}\right) \sin \left(\frac{\lambda \pi}{2}\right) u^{\lambda-1}
\end{aligned}
$$

and

$\epsilon(\alpha, \lambda)=\frac{\mu \lambda \cos \left(\frac{\lambda \pi}{2}\right)+\sqrt{\mu^{2} \lambda^{2} \cos ^{2}\left(\frac{\lambda \pi}{2}\right)+4\left(\lambda^{2}-1\right)\left(\beta+\frac{\delta}{2(1-\alpha)}\right)\left(\gamma-\frac{\delta(1-\alpha)}{2}\right) \sin ^{2}\left(\frac{\lambda \pi}{2}\right)}}{2(\lambda+1)\left(\beta+\frac{\delta}{2(1-\alpha)}\right) \sin \left(\frac{\lambda \pi}{2}\right)}$

then $p \in \mathcal{P}(\alpha)$.

Proof. Define the analytic function $p: \mathbb{D} \rightarrow \mathbb{C}$ as

$$
p(z)=\frac{1+(1-2 \alpha) w(z)}{1-w(z)}
$$

where $w$ is an analytic function in $\mathbb{D}$ with $w(0)=0$. Suppose that there exists a point $z_{0} \in \mathbb{D}$, such that

$$
\operatorname{Re}\{p(z)\}>\alpha \text { for }|z|<\left|z_{0}\right| \text { and } \operatorname{Re}\left\{p\left(z_{0}\right)\right\}=\alpha,
$$


then we have

$$
|w(z)|<1 \text { for }|z|<\left|z_{0}\right| \text { and }\left|w\left(z_{0}\right)\right|=1 .
$$

By Lemma 1 , we have $z_{0} w^{\prime}\left(z_{0}\right)=k w\left(z_{0}\right)$, where $k$ is a real number with $k \geq 1$. Now,

$$
\frac{z_{0} p^{\prime}\left(z_{0}\right)}{2 k(1-\alpha)}=\frac{w\left(z_{0}\right)}{\left(1-w\left(z_{0}\right)\right)^{2}}=\frac{2\left\{\operatorname{Re} w\left(z_{0}\right)-1\right\}}{\left|1-w\left(z_{0}\right)\right|^{4}}
$$

Putting $p\left(z_{0}\right)=\alpha+i y$, we have $w\left(z_{0}\right)=1-\frac{2(1-\alpha)^{2}}{(1-\alpha)^{2}+y^{2}}+i \frac{2(1-\alpha) y}{(1-\alpha)^{2}+y^{2}}$ and

$$
z_{0} p^{\prime}\left(z_{0}\right)=-k \frac{(1-\alpha)^{2}+y^{2}}{2(1-\alpha)}
$$

which is a non positive real number. Also we observe that for the case $0<\lambda<1$

$$
\begin{aligned}
\operatorname{Re} & \left\{\left(p\left(z_{0}\right)-\alpha\right)^{\lambda}\left(\mu+\beta\left(p\left(z_{0}\right)-\alpha\right)+\frac{\gamma}{p\left(z_{0}\right)-\alpha}+\delta \frac{z_{0} p^{\prime}\left(z_{0}\right)}{p\left(z_{0}\right)-\alpha}\right)\right\} \\
= & \operatorname{Re}\left\{\beta\left(p\left(z_{0}\right)-\alpha\right)^{\lambda+1}+\mu\left(p\left(z_{0}\right)-\alpha\right)^{\lambda}+\gamma\left(p\left(z_{0}\right)-\gamma\right)^{\lambda-1}\right. \\
& \left.+\delta z_{0} p^{\prime}\left(z_{0}\right)\left(p\left(z_{0}\right)-\alpha\right)^{\lambda-1}\right\} \\
= & \operatorname{Re}\left\{\beta(i y)^{\lambda+1}+\mu(i y)^{\lambda}+\left(\gamma-k \delta \frac{(1-\alpha)^{2}+y^{2}}{2(1-\alpha)}\right)(i y)^{\lambda-1}\right\} \\
= & \operatorname{Re}\left\{\left(-\beta \sin \frac{\lambda \pi}{2}+i \beta \cos \frac{\lambda \pi}{2}-k \frac{\delta}{2(1-\alpha)}\left(\sin \frac{\lambda \pi}{2}-i \cos \frac{\lambda \pi}{2}\right)\right)|y|^{\lambda+1}\right. \\
& \left.+\mu\left(\cos \frac{\lambda \pi}{2}+i \sin \frac{\lambda \pi}{2}\right)|y|^{\lambda}+\left(\gamma-k \delta \frac{1-\alpha}{2}\right)\left(\sin \frac{\lambda \pi}{2}-i \cos \frac{\lambda \pi}{2}\right)|y|^{\lambda-1}\right\} \\
= & \left(-\beta \sin \frac{\lambda \pi}{2}-k \frac{\delta}{2(1-\alpha)} \sin \frac{\lambda \pi}{2}\right)|y|^{\lambda+1}+\mu\left(\cos \frac{\lambda \pi}{2}\right)|y|^{\lambda} \\
& +\left(\gamma-k \frac{\delta(1-\alpha)}{2}\right)\left(\sin \frac{\lambda \pi}{2}\right)|y|^{\lambda-1} \\
\leq & -\left(\beta+\frac{\delta}{2(1-\alpha)}\right) \sin \frac{\lambda \pi}{2}|y|^{\lambda+1}+\mu \cos \frac{\lambda \pi}{2}|y|^{\lambda}+\left(\gamma-\frac{\delta(1-\alpha)}{2}\right) \sin \frac{\lambda \pi}{2}|y|^{\lambda-1} \\
= & g(|y|, \alpha, \lambda) \leq \max _{u \in(0, \infty)} g(u, \alpha, \lambda)=g(\epsilon(\alpha, \lambda), \alpha, \lambda),
\end{aligned}
$$

which is a contradiction to (1). For the case when $\lambda=1$,

$\operatorname{Re}\left\{\left(p\left(z_{0}\right)-\alpha\right)\left(\mu+\beta\left(p\left(z_{0}\right)-\alpha\right)+\frac{\gamma}{p\left(z_{0}\right)-\alpha}+\delta \frac{z_{0} p^{\prime}\left(z_{0}\right)}{p\left(z_{0}\right)-\alpha}\right)\right\}$ 


$$
\begin{aligned}
& =\operatorname{Re}\left\{\left(p\left(z_{0}\right)-\alpha\right)+\left(\mu+\beta\left(p\left(z_{0}\right)-\alpha\right)+\frac{\gamma}{p\left(z_{0}\right)-\alpha}-\delta k\left(\frac{(1-\alpha)^{2}+y^{2}}{2(1-\alpha) p\left(z_{0}\right)-\alpha}\right)\right)\right\} \\
& \leq \operatorname{Re}\left\{(i y) \mu+\beta(i y)^{2}+\gamma-\delta\left(\frac{(1-\alpha)^{2}+y^{2}}{2(1-\alpha)}\right)\right\} \\
& =-\left(\beta+\frac{\delta}{2(1-\alpha)}\right) y^{2}+\gamma-\delta \frac{(1-\alpha)}{2} \\
& \leq \gamma-\delta \frac{(1-\alpha)}{2}=g(\epsilon(\alpha, 1), \alpha, 1) .
\end{aligned}
$$

This contradicts (1). Hence the proof.

Remark 1 By taking $\mu=\delta=1$ and $\beta=\gamma=0$ in Theorem 1, we get the result obtained in [6, Theorem 2.29].

By taking $p(z)=z f^{\prime}(z) / f(z)$ and $\alpha=0$ in Theorem 1, we have the following result:

Corollary 1 For a function $\mathrm{f} \in \mathcal{A}$ and $0<\lambda \leq 1$, if

$$
\begin{aligned}
\operatorname{Re}\left\{(\beta-\delta)\left(\frac{z f^{\prime}(z)}{f(z)}\right)^{\lambda+1}+\left(\mu+\delta+\delta \frac{z f^{\prime \prime}(z)}{f^{\prime}(z)}\right)\left(\frac{z f^{\prime}(z)}{f(z)}\right)^{\lambda}\right. & \left.+\gamma\left(\frac{z f^{\prime}(z)}{f(z)}\right)^{\lambda-1}\right\} \\
& >g(\epsilon(0, \lambda), 0, \lambda),
\end{aligned}
$$

then $f \in \mathcal{S}^{*}$.

Theorem 2 For an analytic function $\mathrm{p}$ in $\mathbb{D}$ with $\mathrm{p}(0)=1$ and $0 \leq \alpha<1$, if

$$
\frac{z p^{\prime}(z)}{(p(z)-\alpha)^{1 / \beta}} \neq i \delta
$$

for all $\delta \in \mathbb{R}$, with $|\delta| \geq 1$ and $\beta=\frac{1}{2 n-1}, n \in \mathbb{N}$, then $p \in \mathcal{P}(\alpha)$.

Proof. Let

$$
h(z)=\left(\frac{p(z)-\alpha}{1-\alpha}\right)^{1 / \beta} .
$$

We note that $h$ is analytic in $\mathbb{D}$, with $h(0)=1$. Here $p(z) \neq \alpha$ for $z \in \mathbb{D}$, suppose that there exist a point $z_{1} \in \mathbb{D}$ such that $p\left(z_{1}\right)=\alpha$, then $z_{1}$ is a zero of multiplicity $m \geq 1$ such that

$$
h(z)=\left(z-z_{1}\right)^{m} g(z) \quad(m \in \mathbb{N}),
$$


where $g(z)$ is analytic in $\mathbb{D}$ and $g\left(z_{1}\right) \neq 0$. Therefore we have

$$
\frac{z p^{\prime}(z)}{(p(z)-\alpha)}=\beta\left(\frac{m z}{z-z_{1}}+\frac{z g^{\prime}(z)}{g(z)}\right) \text {. }
$$

But the imaginary part of right-hand side of $(7)$ can take any value when $z$ approaches $z_{1}$. This contradicts (6). Therefore $p(z) \neq \alpha$, that is $h(z) \neq 0$. Suppose that there exist a point $z_{0} \in \mathbb{D}$ such that

$$
\operatorname{Re}\left\{(h(z))^{\beta}\right\}>0 \text { for }|z|<\left|z_{0}\right| \text { and } \operatorname{Re}\left\{\left(h\left(z_{0}\right)\right)^{\beta}\right\}=0 \quad\left(h\left(z_{0}\right) \neq 0\right) .
$$

Setting

$$
\phi(z)=\frac{1-(h(z))^{\beta}}{1+(h(z))^{\beta}},
$$

we observe that

$$
|\phi(z)|<1 \text { for }|z|<\left|z_{0}\right|,\left|\phi\left(z_{0}\right)\right|=1 \text { and } \phi(0)=0 .
$$

Hence the conditions of Lemma 1 are satisfied. By taking

$$
\left(\mathrm{h}\left(z_{0}\right)\right)^{\beta}=i \mathrm{y},
$$

where $\mathrm{y}$ is a non zero positive real number, and by using Lemma 1, we obtain

$$
\frac{z_{0} \phi^{\prime}\left(z_{0}\right)}{\phi\left(z_{0}\right)}=\frac{-2 \beta\left(h\left(z_{0}\right)\right)^{\beta-1} z_{0} h^{\prime}\left(z_{0}\right)}{1-(h(z))^{2 \beta}}=k,
$$

and

$$
-z_{0} h^{\prime}\left(z_{0}\right)=\frac{k\left(1+y^{2}\right)}{2 \beta\left(h\left(z_{0}\right)\right)^{-1}(\mathfrak{i y})} .
$$

Now,

$$
\begin{aligned}
\frac{z_{0} p^{\prime}\left(z_{0}\right)}{\left(p\left(z_{0}\right)-\alpha\right)^{1 / \beta}} & =\frac{-k(1-\alpha)^{1-(1 / \beta)}\left(1+y^{2}\right)}{2\left((i y)^{1 / \beta}\right.} \\
& =\frac{-k(1-\alpha)^{1-(1 / \beta)}\left(1+y^{2}\right)}{2 y^{1 / \beta}}\left(\cos \frac{\pi}{2 \beta}-i \sin \frac{\pi}{2 \beta}\right) .
\end{aligned}
$$

For $\beta=\frac{1}{2 n-1}, n \in \mathbb{N}$

$$
\frac{z_{0} p^{\prime}\left(z_{0}\right)}{\left(p\left(z_{0}\right)-\alpha\right)^{1 / \beta}}=\frac{-k(1-\alpha)^{1-(1 / \beta)}\left(1+y^{2}\right)}{2 y^{1 / \beta}}(-1)^{n+1} i=i \delta, \quad \delta \in \mathbb{R},
$$


which is a contradiction to (6), where

$$
\begin{aligned}
|\delta| & =\left|\frac{k(1-\alpha)^{1-(1 / \beta)}\left(1+y^{2}\right)(-1)^{n+2}}{2 y^{1 / \beta}}\right| \\
& \geq \frac{(1-\alpha)^{1-(1 / \beta)}\left(1+y^{2}\right)}{2 y^{1 / \beta}} \geq 1 .
\end{aligned}
$$

Hence the proof.

For the choice of $p(z)=z f^{\prime}(z) / f(z), \beta=1$ and $p(z)=f^{\prime}(z), \beta=1$ and $\alpha=0$, in Theorem 2, we have the following Corollary 2 and Corollary 3 respectively.

Corollary 2 For $0 \leq \alpha<1$, if the function $\mathrm{f} \in \mathcal{A}$ satisfies

$$
\frac{z f^{\prime}(z)\left(1-\frac{z f^{\prime}(z)}{f(z)}\right)+z^{2} f^{\prime \prime}(z)}{z f^{\prime}(z)-\alpha f(z)} \neq i \delta \quad(\delta \in \mathbb{R},|\delta| \geq 1)
$$

then $f \in \mathcal{S}^{*}(\alpha)$.

Corollary 3 If $\mathrm{f} \in \mathcal{A}$ satisfies

$$
\frac{z f^{\prime \prime}(z)}{f^{\prime}(z)} \neq i \delta \quad(\delta \in \mathbb{R},|\delta| \geq 1)
$$

then $\mathrm{f}$ is univalent.

Theorem 3 Let $\alpha, \beta, \gamma, \delta \in \mathbb{R}$ with $0 \leq \alpha<1, \gamma<\frac{\beta+\delta}{2}$ and let $\mathrm{G}(z)$ be a complex valued function defined in $\mathbb{D}$. If $\mathrm{p}$ is analytic in $\mathbb{D}$ with $\mathrm{p}(0)=1$ and

$$
\begin{aligned}
& \operatorname{Re}\left\{\gamma z^{3} p^{\prime \prime \prime}(z)+(3 \gamma+\beta) z^{2} p^{\prime \prime}(z)+(\gamma+2 \beta+\delta) z p^{\prime}(z)+G(z) p(z)\right\} \\
& \quad>\mu(\alpha, \beta, \gamma, \delta, G(z)),
\end{aligned}
$$

where

$$
\mu(\alpha, \beta, \gamma, \delta, G(z))=\frac{(1-\alpha)[\operatorname{Im}(G(z))]^{2}-[\delta+\beta-2 \gamma]^{2}}{2(\delta+\beta-2 \gamma)}+\alpha \operatorname{Re}\{\mathrm{G}(z)\},
$$

then $p \in \mathcal{P}(\alpha)$. 
Proof. Let the function $p$ be defined as in (2), suppose that there exists a point $z_{0}$ in $\mathbb{D}$ satisfying (3). By defining $h: \mathbb{D} \rightarrow \mathbb{C}$ as $h(z)=(1+(1-2 \alpha) z) /(1-z)$, we have $p \nprec h$. By Lemma 2, there exist a $\zeta_{0} \in \partial \mathbb{D}$ and $m \geq 1$ such that

$$
\operatorname{Re}\left\{1+\frac{z_{0} p^{\prime \prime}\left(z_{0}\right)}{p^{\prime}\left(z_{0}\right)}\right\} \geq m \operatorname{Re}\left\{1+\frac{\zeta_{0} h^{\prime \prime}\left(\zeta_{0}\right)}{h^{\prime}\left(\zeta_{0}\right)}\right\}=0
$$

and

$$
\operatorname{Re}\left\{\frac{z_{0}^{2} \mathrm{p}^{\prime \prime \prime}\left(z_{0}\right)}{\mathrm{p}^{\prime}\left(z_{0}\right)}\right\} \geq \mathrm{m}^{2} \operatorname{Re}\left\{\frac{\zeta_{0}^{2} \mathrm{~h}^{\prime \prime \prime}\left(\zeta_{0}\right)}{\mathrm{h}^{\prime}\left(\zeta_{0}\right)}\right\}>0 .
$$

Using (5) and (9), we obtain

$$
\operatorname{Re}\left\{z_{0}^{2} \mathrm{p}^{\prime \prime}\left(z_{0}\right)\right\} \leq-z_{0} \mathrm{p}^{\prime}\left(z_{0}\right) \text { and } \operatorname{Re}\left\{z_{0}^{3} \mathrm{p}^{\prime \prime \prime}\left(z_{0}\right)\right\} \leq 0 .
$$

From (5), (10) and by taking $p(z)=\alpha+i y(y \in \mathbb{R})$, we have the following inequality

$$
\begin{aligned}
& \operatorname{Re}\left\{\gamma z_{0}^{3} p^{\prime \prime \prime}\left(z_{0}\right)+(3 \gamma+\beta) z_{0}^{2} p^{\prime \prime}\left(z_{0}\right)+(\gamma+2 \beta+\delta) z_{0} p^{\prime}\left(z_{0}\right)+G(z) p\left(z_{0}\right)\right\} \\
& \leq(\delta+\beta-2 \gamma) z_{0} p^{\prime}\left(z_{0}\right)+\alpha \operatorname{Re}\left\{G\left(z_{0}\right)\right\}-\operatorname{Im}\left\{G\left(z_{0}\right)\right\} y \\
& \leq-(\delta+\beta-2 \gamma)\left(\frac{(1-\alpha)^{2}+y^{2}}{2(1-\alpha)}\right)+\alpha \operatorname{Re}\left\{G\left(z_{0}\right)\right\}-\operatorname{Im}\left\{G\left(z_{0}\right)\right\} y \\
& \leq \frac{(1-\alpha)\left(\operatorname{Im}\left\{G\left(z_{0}\right)\right\}\right)^{2}-(\delta+\beta-2 \gamma)^{2}}{2(\delta+\beta-2 \gamma)}+\alpha \operatorname{Re}\left\{G\left(z_{0}\right)\right\} \\
& =\mu\left(\alpha, \beta, \gamma, \delta, G\left(z_{0}\right)\right),
\end{aligned}
$$

which contradicts (8) and completes the proof.

On taking $\alpha=\beta=\gamma=0, \delta=1$ and $\mathrm{G}(z) \equiv 1$ in Theorem 3 , we get the following Corollary that improves the result of Miller [7, p.80].

Corollary 4 For an analytic function $\mathrm{p}$ in $\mathbb{D}$ with $\mathrm{p}(0)=1$, if

$$
\operatorname{Re}\left\{p(z)+z p^{\prime}(z)\right\}>-\frac{1}{2},
$$

then $p \in \mathcal{P}$.

By taking $\gamma=\beta=0, \mathrm{G}(z) \equiv 1$ and $\gamma=\beta=0, \delta=1, \mathrm{G}(z) \equiv 1$ in Theorem 3, we obtain the Corollary 5 and Corollary 6 respectively, which are due to Kim et al. [6, Theorem 2.6]. 
Corollary 5 For an analytic function $p$ in $\mathbb{D}$ with $\mathrm{p}(0)=1$, if

$$
\operatorname{Re}\left\{\delta z p^{\prime}(z)+p(z)\right\}>\alpha-\frac{(1-\alpha) \delta}{2}, \quad(0 \leq \alpha<1)
$$

then $p \in \mathcal{P}(\alpha)$.

Corollary 6 For an analytic function $\mathrm{p}$ in $\mathbb{D}$ with $\mathrm{p}(0)=1$, if

$$
\operatorname{Re}\left\{z p^{\prime}(z)+p(z)\right\}>\frac{(3 \alpha-1)}{2}, \quad(0 \leq \alpha<1)
$$

then $p \in \mathcal{P}(\alpha)$.

Theorem 4 Let $\mathrm{p}$ be an analytic function in $\mathbb{D}$, with $\mathrm{p}(0)=1$ for $\beta>0$, if

$$
\left|\operatorname{Im}\left\{(p(z))^{1 / \beta}+\frac{z p^{\prime}(z)}{p(z)}\right\}\right|<\frac{1}{2\left|(p(z))^{1 / \beta}\right|}\left((2-\beta)\left|(p(z))^{2 / \beta}\right|-\beta\right),
$$

then $(p(z))^{1 / \beta} \in \mathcal{P}$.

Proof. Define the function $p: \mathbb{D} \rightarrow \mathbb{C}$ as

$$
p(z)=\left(\frac{1+w(z)}{1-w(z)}\right)^{\beta}
$$

or equivalently

$$
w(z)=\frac{p(z)^{1 / \beta}-1}{p(z)^{1 / \beta}+1}
$$

then $w$ is analytic in $\mathbb{D}$ with $w(0)=0$. Suppose that there exist a point $z_{0}$ in $\mathbb{D}$ such that

$$
\operatorname{Re}\left\{(p(z))^{1 / \beta}\right\}>0 \text { for }|z|<\left|z_{0}\right| \text { and } \operatorname{Re}\left\{\left(p\left(z_{0}\right)\right)^{1 / \beta}\right\}=0,
$$

we obtain

$$
|w(z)|<1 \text { for }|z|<\left|z_{0}\right| \text { and }\left|w\left(z_{0}\right)\right|=1 .
$$

Therefore by using Jack's Lemma, a simple calculation yields

$$
\frac{1}{\beta} \frac{z_{0} p^{\prime}\left(z_{0}\right)}{p\left(z_{0}\right)}=\frac{2 z_{0} w^{\prime}\left(z_{0}\right)}{1-\left(w\left(z_{0}\right)\right)^{2}}=\frac{2 k w\left(z_{0}\right)}{1-\left(w\left(z_{0}\right)\right)^{2}} .
$$

Hence

$$
\frac{1}{2 k \beta} \frac{z_{0} p^{\prime}\left(z_{0}\right)}{p\left(z_{0}\right)}=\frac{w\left(z_{0}\right)}{1-\left(w\left(z_{0}\right)\right)^{2}} .
$$


On taking $\left(p\left(z_{0}\right)\right)^{1 / \beta}=i y$, where $y$ is a nonzero real number, we obtain

$$
w\left(z_{0}\right)=\frac{y^{2}-1}{1+y^{2}}+i \frac{2 y}{1+y^{2}}
$$

and

$$
\frac{z_{0} p^{\prime}\left(z_{0}\right)}{p\left(z_{0}\right)}=\frac{2 k \beta w\left(z_{0}\right)}{1-\left(w\left(z_{0}\right)\right)^{2}}
$$

where $k$ is a real number, with $k \geq 1$. Therefore

$$
\begin{aligned}
\left|\operatorname{Im}\left\{\left(p\left(z_{0}\right)\right)^{1 / \beta}+\frac{z_{0} p^{\prime}\left(z_{0}\right)}{p\left(z_{0}\right)}\right\}\right| & =\left|\operatorname{Im}\left\{\left(p\left(z_{0}\right)\right)^{1 / \beta}\right\}+\operatorname{Im}\left\{\frac{z_{0} p^{\prime}\left(z_{0}\right)}{p\left(z_{0}\right)}\right\}\right| \\
& =\left|y+2 k \beta \frac{\left(1+y^{2}\right)}{4 y}\right| \\
& \geq\left|y+\beta \frac{\left(1+y^{2}\right)}{2 y}\right| \\
& \geq\left|y-\beta \frac{\left(1+y^{2}\right)}{2 y}\right| \geq|y|-\beta \frac{\left(1+|y|^{2}\right)}{2|y|} \\
& =\left|\left(p\left(z_{0}\right)\right)^{1 / \beta}\right|-\frac{\beta\left(1+\left|\left(p\left(z_{0}\right)\right)^{2 / \beta}\right|\right)}{2\left|\left(p\left(z_{0}\right)\right)^{1 / \beta}\right|},
\end{aligned}
$$

which contradicts (11). Hence the proof.

Theorem 5 For an analytic function $\mathrm{p}$ in $\mathbb{D}$ with $\mathrm{p}(0)=1$, if $\mathrm{p}$ satisfies

$$
\operatorname{Re}\left\{(p(z))^{2}+\frac{z p^{\prime}(z)}{p(z)}\right\}<1-c-\frac{c}{2(1-c)} \quad(0<c<1),
$$

then $\mathrm{p} \in \mathcal{P}$. Also $\mathrm{p}(z) \prec \sqrt{1+\mathrm{cz}}$.

Proof. Define a function $p: \mathbb{D} \rightarrow \mathbb{C}$ by

$$
\begin{aligned}
p(z) & =\sqrt{1+c w(z)}, \quad(z \in \Delta) \\
& =1+p_{1} z+p_{2} z^{2}+\ldots,
\end{aligned}
$$

or equivalently

$$
w(z)=\frac{p^{2}(z)-1}{c}=w_{1} z+w_{2} z^{2}+\ldots
$$


we observe that $w$ is analytic in $\mathbb{D}$ and $w(0)=0$.

Suppose that there exist a point $z_{0}$ in $\mathbb{D}$, such that

$$
\operatorname{Re}\{p(z)\}>0 \text { for }|z|<\left|z_{0}\right| \text { and } \operatorname{Re}\left\{p\left(z_{0}\right)\right\}=0
$$

and

$$
\max |w(z)|=\left|w\left(z_{0}\right)\right|=1 \quad|z| \leq\left|z_{0}\right| .
$$

By Lemma 1, there exist a number $k \geq 1$ such that $z_{0} w^{\prime}\left(z_{0}\right)=k w\left(z_{0}\right)$. Without loss of generality we may assume that $w\left(z_{0}\right)=e^{i \theta}$, where $\theta \in[-\pi, \pi]$, for this $z_{0}$, we have

$$
\begin{aligned}
\operatorname{Re}\left\{\left(\mathrm{p}\left(z_{0}\right)\right)^{2}+\frac{z_{0} p^{\prime}\left(z_{0}\right)}{p\left(z_{0}\right)}\right\} & =\operatorname{Re}\left\{1+c w\left(z_{0}\right)\right\}+\operatorname{Re}\left\{\frac{c k w\left(z_{0}\right)}{2\left(1+c w\left(z_{0}\right)\right.}\right\} \\
& =\operatorname{Re}\left\{1+c e^{i \theta}\right\}+\frac{c k}{2} \operatorname{Re}\left\{\frac{e^{i \theta}}{1+c e^{i \theta}}\right\} \\
& =\operatorname{Re}\{1+c \cos \theta+i \sin \theta\}+\frac{c k}{2} \mathcal{R e}\left\{\frac{\cos \theta+\sin \theta}{1+c e^{i \theta}}\right\} \\
& \geq 1+c \cos \theta+\frac{c}{2}\left(\frac{\cos \theta+c}{1+c^{2}+2 c \cos \theta}\right)=H(\cos \theta) .
\end{aligned}
$$

Let $t=\cos \theta$ then

$$
H(t)=1+c t+\frac{c}{2}\left(\frac{t+c}{1+c^{2}+2 c t}\right) .
$$

Since $H(t)$ is an increasing function,

$$
\begin{aligned}
H(t) \geq H(-1) & =1-c+\frac{c}{2}\left(\frac{c-1}{1+c^{2}-2 c}\right) \\
& =1-c-\frac{c}{2}\left(\frac{1-c}{(1-c)^{2}}\right) \\
& =1-c-\frac{c}{2(1-c)},
\end{aligned}
$$

which is a contradiction to (12) and implies that, $\operatorname{Re}\{p(z)\}>0$ and $\left|w\left(z_{0}\right)\right|<1$. That is $\mathrm{p}(z) \prec \sqrt{1+\mathrm{c} z}$ and $\mathrm{p} \in \mathcal{P}, z \in \mathbb{D}$.

Following results are obtained as the consequence of Theorem 5 .

For the choice of $p(z)=\frac{z f^{\prime}(z)}{f(z)}$ in Theorem 5, we have the following: 
Corollary 7 If $\mathrm{f} \in \mathcal{A}$ satisfies

$$
\operatorname{Re}\left\{\left(\frac{z f^{\prime}(z)}{f(z)}\right)^{2}+\frac{z f^{\prime \prime}(z)}{f^{\prime}(z)}-\frac{z f^{\prime}(z)}{f(z)}+1\right\}<(1-c)-\frac{c}{2(1-c)} \quad(0<c<1) \text {, }
$$

then $\mathrm{f} \in \mathcal{S}^{*}\left(\mathrm{q}_{\mathrm{c}}\right)$.

By taking $p(z)=\frac{z \sqrt{f^{\prime}(z)}}{f(z)}$ in Theorem 5, we have the following:

Corollary 8 If $\mathrm{f} \in \mathcal{A}$ satisfies

$$
\operatorname{Re}\left\{\frac{z^{2} f^{\prime}(z)}{(f(z))^{2}}+\frac{z f^{\prime \prime}(z)}{2 f^{\prime}(z)}-\frac{z f^{\prime}(z)}{f(z)}+1\right\}<(1-\lambda)-\frac{\lambda}{2(1-\lambda)} \quad(0<\lambda<1),
$$

then $\mathrm{f} \in \mathcal{U}(\lambda)$ and hence it is univalent.

\section{Acknowledgement}

The authors are thankful to the referee for the insightful suggestions.

\section{References}

[1] J. A. Antonino, S. S. Miller, Third-order differential inequalities and subordinations in the complex plane, Complex Var. Elliptic Equ., 56 (5) (2011), 439-454.

[2] P. L. Duren, Univalent functions, Grundlehren der Mathematischen Wissenschaften, 259, Springer-Verlag, New York, 1983.

[3] Huo Tang, H. M. Srivastava, Shu-Hai Li, Li-Na Ma, Third-order differential subordination and superordination results for meromorphically multivalent functions associated with the Liu-Srivastava operator, Abstr. Appl. Anal., 2014, Art. ID 792175, 11 pp.

[4] I. S. Jack, Functions starlike and convex of order $\alpha$, J. London Math. Soc., 3 (2) (1971), 469-474.

[5] I. H. Kim, N. E. Cho, Sufficient conditions for Carathéodory functions, Comput. Math. Appl., 59 (6) (2010), 2067-2073.

[6] I. H. Kim, Y. J. Sim, N. E. Cho, New criteria for Carathéodory functions, J. Inequal. Appl., 13 (2019), 16 pp. 
[7] S. Miller, Differential inequalities and Carathéodory functions, Bull. Amer. Math. Soc., 81 (1975), 79-81.

[8] S. S. Miller, P. T. Mocanu, Differential subordinations and univalent functions, Michigan Math. J., 28 (2) (1981), 157-172.

[9] S. S. Miller, P. T. Mocanu, Differential subordinations, Monographs and Textbooks in Pure and Applied Mathematics, 225, Marcel Dekker, Inc., New York, 2000.

[10] S. S. Miller, P. T. Mocanu, M. O. Reade, All $\alpha$-convex functions are starlike, Rev. Roumaine Math. Pures Appl., 17 (1972), 1395-1397.

[11] N. Xu, Sufficient conditions and applications for Carathéodory functions, J. Math., 2013, Art. ID 930290, 4 pp.

[12] M. Nunokawa, Differential inequalities and Carathéodory functions, Proc. Japan Acad. Ser. A Math. Sci., 65 (10) (1989), 326-328.

[13] M. Nunokawa, A. Ikeda, N. Koike, Y. Ota, H. Saitoh, Differential inequalities and Carathéodory functions, J. Math. Anal. Appl., 212 (1) (1997), $324-332$.

[14] M. Nunokawa, S. Owa, N. Takahashi, H. Saitoh, Sufficient conditions for Carathéodory functions, Indian J. Pure Appl. Math., 33 (9) (2002), $1385-1390$.

[15] M. Nunokawa, M. Obradović, S. Owa, One criterion for univalency, Proc. Amer. Math. Soc., 106 (4) (1989), 1035-1037.

[16] S. Ponnusamy, P. Vasundhra, Starlikeness of nonlinear integral transforms, J. Anal., 15 (2007), 195-210.

[17] J. Sokół, On some subclass of strongly starlike functions, Demonstratio Math., 31 (1) (1998), 81-86.

[18] J. Sokół, J. Stankiewicz, Radius of convexity of some subclasses of strongly starlike functions, Zeszyty Nauk. Politech. Rzeszowskiej, 19 (1996), 101105. 